J. Austral. Math. Soc. 19 (Series B), (1976), 493-512.

\title{
ON THE ASYMPTOTIC SOLUTION OF AN ELLIPTIC INTERIOR LAYER PROBLEM
}

\author{
N. G. BARTON \\ (Received 21 November 1975) \\ (Revised 16 August 1976)
}

\begin{abstract}
An interior layer problem posed by an elliptic partial differential equation of the type $\varepsilon \nabla^{2} \phi-x \partial \phi / \partial y=f(x, y, \varepsilon), 0<\varepsilon \ll 1$, is investigated. This equation arises, for example, in the theory of rotating fluids and the important feature of the problem is an interior layer of width $O\left(\varepsilon^{1 / 3}\right)$ in which the solution has a relatively large magnitude.

The paper considers the simplest case which involves an interior layer, that is, where the domain is rectangular and $f(x, y, \varepsilon)=\varepsilon A$ for $\boldsymbol{A}$ constant. $A$ leading approximation is derived and it is shown to be asymptotic to the exact solution in nearly all of the domain as $\varepsilon \rightarrow 0$. The error estimates are derived using an a priori estimate for the solution of elliptic equations and a technique which optimizes the estimates is introduced. The applicability and lımitations of the estimation technique are discussed briefly.
\end{abstract}

\section{Introduction}

In this paper, a leading approximation $\psi$ to the solution $\phi$ of the singular perturbation problem

$$
\begin{gathered}
\varepsilon\left\{\frac{\partial^{2} \phi}{\partial x^{2}}+\frac{\partial^{2} \phi}{\partial y^{2}}\right\}-x \frac{\partial \phi}{\partial y}=\varepsilon A \text { in } G, \\
\left.\phi\right|_{\partial G}=\phi^{*}(x, y, \varepsilon)
\end{gathered}
$$

is constructed, and error bounds on $\psi$ are established as $\varepsilon \rightarrow 0$. The domain $G$ is the rectangle $-a<x<a,-\frac{1}{2}<y<\frac{1}{2}$ whose boundary $\partial G$ consists of the segments denoted $\left\{\gamma_{1}\right\}_{i=1}^{6}$ in figure 1 and $A$ is a constant. For $x>0$, the boundary value $\phi^{*}$ is 


$$
\phi^{*}(x, y, \varepsilon)= \begin{cases}0 & \text { on } \gamma_{1} \\ \varepsilon b_{2}(y) & \text { on } \gamma_{2} \\ \varepsilon b_{3}(x) & \text { on } \gamma_{3}\end{cases}
$$

and it is assumed, without a serious loss of generality, that $\phi^{*}$ has the symmetry

$$
\phi^{*}(x, y, \varepsilon)=\phi^{*}(-x,-y, \varepsilon), \quad(x, y) \in \partial G
$$

The boundary value $\phi^{*}$ is assumed to be sufficiently smooth for the problem to have a unique, twice continuously differentiable solution.

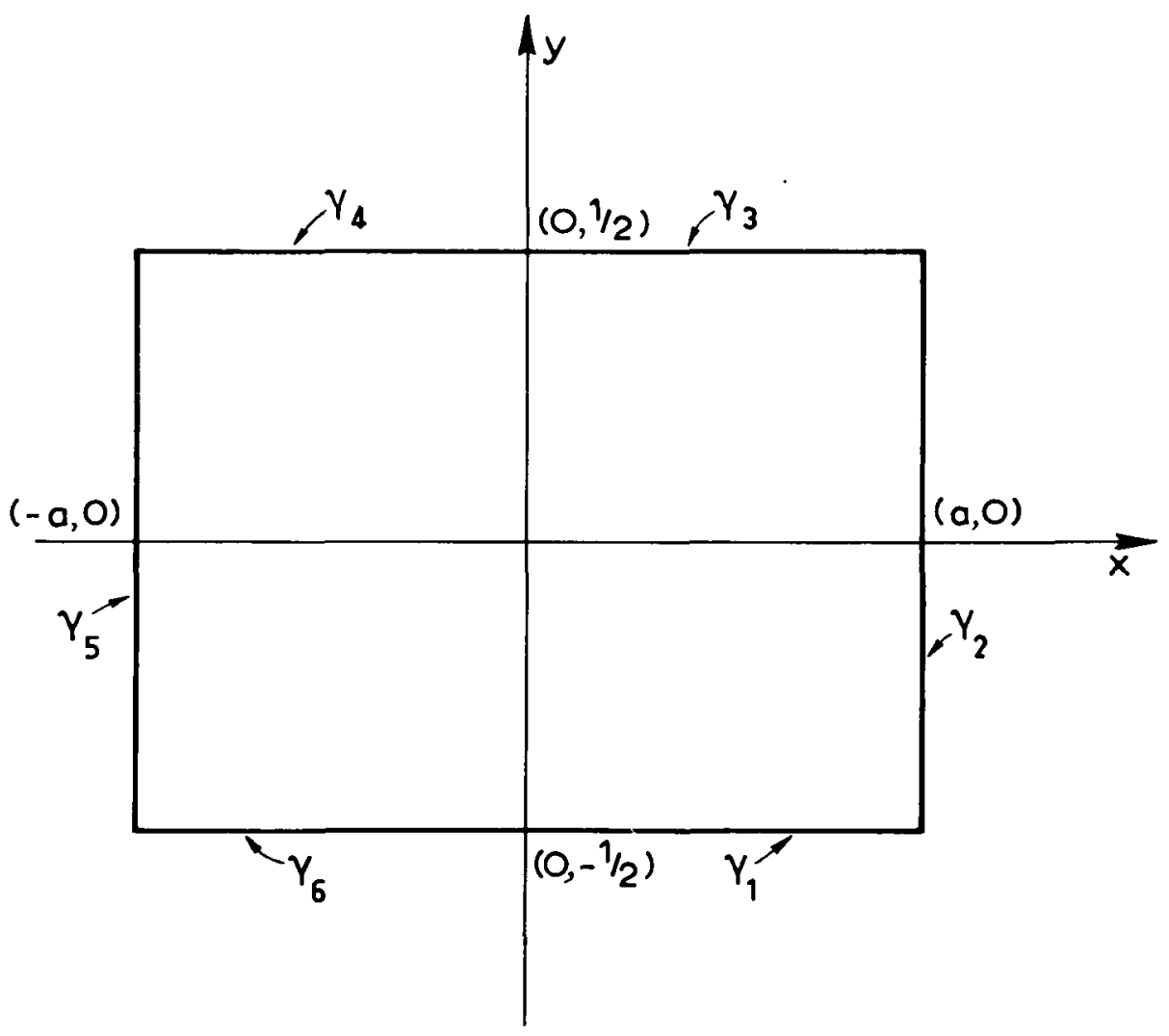

Figure 1.

Illustrating the doman $G$ and the boundary $U_{i-1}^{i} \gamma$,

This symmetric problem with constant inhomogeneous term is the simplest model which includes an interior layer inside the domain $G$ as well as 
distinct boundary layers at $\partial G$. The assumed scaling in the inhomogeneous term of (1.1) and in the boundary conditions (1.3) is consistent and is convenient to demonstrate the magnitude of the solution in various regions. These choices are only made for simplicity and the essential features of the problem are unchanged for more general forcing terms and boundary conditions.

The approximation $\psi$ is found to possess an interior layer of width $O\left(\varepsilon^{1 / 3}\right)$ centred on the line $x=0$ and, in this layer, $\psi$ has the relatively large magnitude $O\left(\varepsilon^{2 / 3}\right)$ compared to the magnitude $O(\varepsilon)$ elsewhere in $G$. Interior layer problems of this sort are known to occur in the study of rotating fluids (see references $[3,8]$ ) and particularly in geophysical fluid dynamics (references $[6,16,17,18])$. Interior layers have previously been studied as elliptic singular perturbation problems by Eckhaus [15] and Cook \& Ludford [10] who, respectively, considered the effect of non-convex boundaries and of discontinuous boundary data. The solution of $(1.1,1.2)$ also possesses boundary layers adjacent to segments of $\partial G$ and, in this respect, the problem resembles previous elliptic singular perturbation problems described in references $[7,10,11,13,14]$.

An estimate of the error in $\psi$ is obtained by observing that the error function $w$,

$$
w=\phi-\psi
$$

satisfies an elliptic problem with an inhomogeneous (or forcing) term depending on the construction of $\psi$, that is, for a particular $g(x, y, \varepsilon)$,

$$
\begin{gathered}
\varepsilon\left\{\frac{\partial^{2} w}{\partial x^{2}}+\frac{\partial^{2} w}{\partial y^{2}}\right\}-x \frac{\partial w}{\partial y}=\varepsilon g(x, y, \varepsilon) \text { in } G, \\
\left.w\right|_{\partial G}=0 .
\end{gathered}
$$

An upper bound for the magnitude of $w$ is then obtained by applying an $a$ priori estimate for solutions of two-dimensional elliptic equations, (see Barton [4]). This gives a pointwise estimate for the error, that is, for the norm $\|w\|_{C(G)}$ where

$$
\|w\|_{C(G)}=\sup _{G}|w| .
$$

An interesting feature of the estimation technique is the smoothing of the approximation by $C^{\infty}$ mollifiers near the ends of the interior layer. This procedure is necessary for the use of the a priori estimate and the optimum size of the mollified region is calculated. A discussion of the results and the applicability of the estimation technique is given in sections 3,4 . 


\section{Construction of a uniform approximation}

A leading approximation to the solution of $(1.1,1.2)$ is constructed for $x>0$ in this section. For $x<0$, the approximation is given by the symmetry property

$$
\phi(x, y, \varepsilon)=\phi(-x,-y, \varepsilon), \quad(x, y) \in G
$$

which holds provided the boundary value $\phi^{*}$ satisfies (1.4). The construction of an approximation is quite complicated since there are three boundary layer regions to be considered. Two of these regions (indicated by III and IV in figure 2) are well-understood and have been studied in detail for example by Eckhaus \& de Jager [14]. The other boundary layer region is the interior layer (denoted by II in Figure 2) and it is the main concern of the present paper.

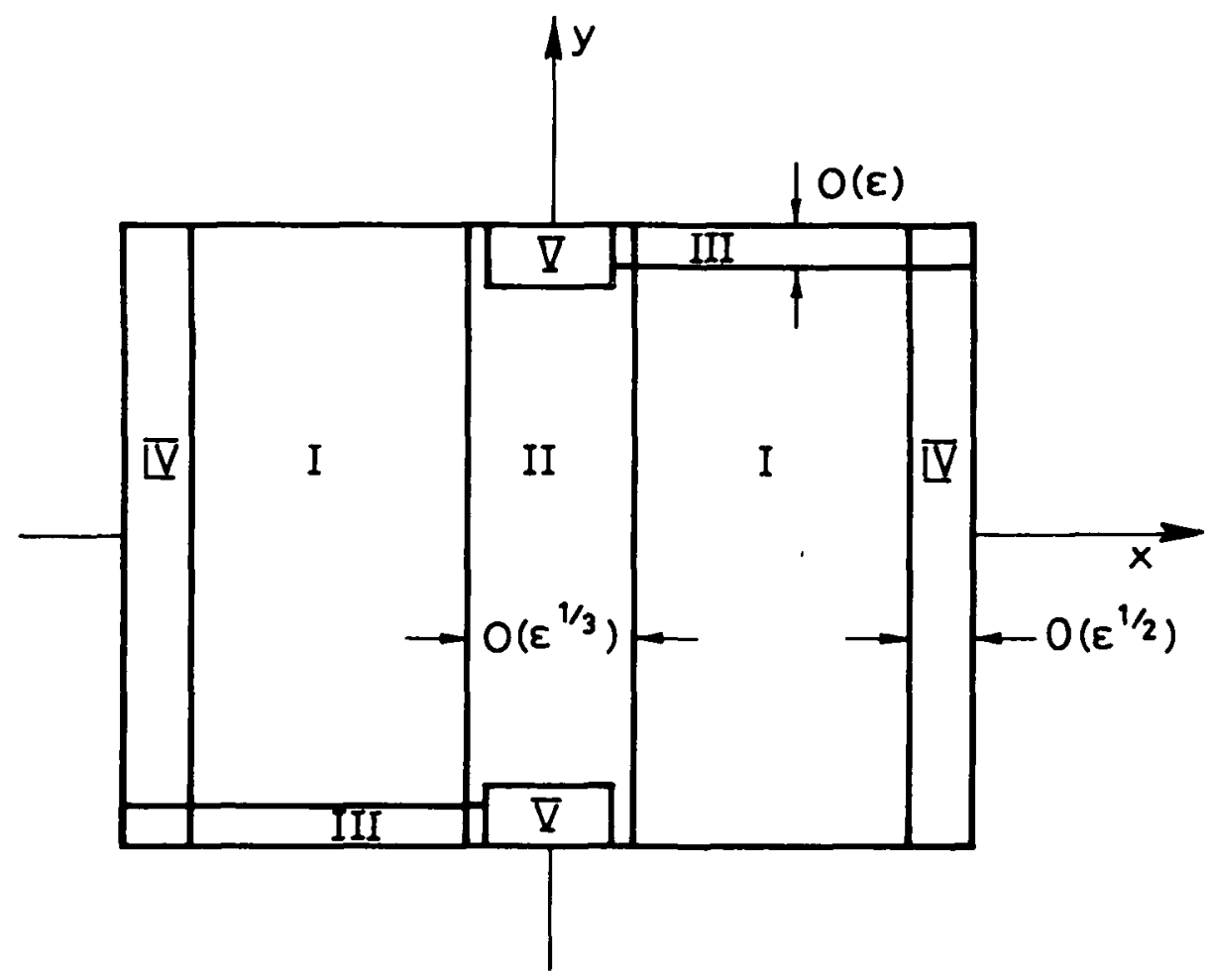

Figure 2.

Illustrating the various regions in $G$. The numbers I to $V$ denote, respectively, the outer region, the interior layer, the ordinary boundary layer, the parabolic boundary layer and the 'corner' regions. 


\section{The outer region}

A suitable Poincaré expansion for $\psi$ in this region is

$$
\psi^{1}(x, y, \varepsilon)=\sum_{n=0}^{\infty} \varepsilon^{1+n} \psi_{n}^{\prime}(x, y)
$$

where the $\left\{\psi_{n}^{1}\right\}$ satisfy the first order equations

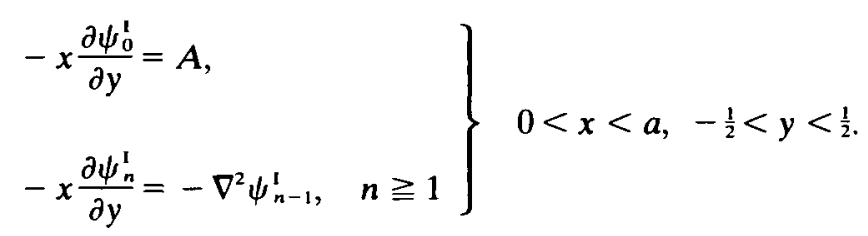

Clearly, the $\left\{\psi_{n}^{1}\right\}$ can only satisfy a boundary condition at $y=+\frac{1}{2}$ or $-\frac{1}{2}$ with a boundary layer occurring at the other end of the lines $x=$ constant. This question of boundary layer placement has been resolved by Eckhaus \& de Jager [14] whose results imply that the boundary conditions on the $\left\{\psi_{n}^{1}\right\}$ are to be applied at $\gamma_{1},\left(y=-\frac{1}{2}\right)$.

The leading outer approximation $\varepsilon \psi_{0}^{1}(x, y)$ is readily found to be

$$
\varepsilon \psi_{0}^{\prime}(x, y)=-\varepsilon A\left(y+\frac{1}{2}\right) x^{-1}, \quad 0<\varepsilon \ll 1 .
$$

This term becomes singular as $x \rightarrow 0$, and a Poincaré expansion of the form (2.2) is clearly unsuitable throughout the whole of $G$. This leads to a consideration of the interior layer.

\section{The interior layer}

An examination of (1.1) shows that $\varepsilon \partial^{2} \phi / \partial x^{2}$ serves to balance $-x \partial \phi / \partial y$ near $x=0$, and it follows that the interior layer has thickness $O\left(\varepsilon^{1 / 3}\right)$. A suitable expansion for $\psi$ in this region is

$$
\psi^{\prime \prime}(x, y, \varepsilon)=\sum_{n=0}^{\infty} \varepsilon^{2(1+n) / 3} \psi_{n}^{\prime \prime}(\xi, \eta)
$$

where $\xi$ is defined by the stretching transformation

$$
\xi=\varepsilon^{-1 / 3} x, \quad x>0
$$

and

$$
\eta=\frac{1}{2}+y \text {. }
$$

The $\left\{\psi_{n}^{\prime \prime}\right\}$ are the solutions of the equations

$$
\frac{\partial^{2} \psi_{0}^{\mathrm{II}}}{\partial \xi^{2}}-\xi \frac{\partial \psi_{0}^{\mathrm{II}}}{\partial \eta}=A
$$




$$
\frac{\partial^{2} \psi_{n}^{\prime \prime}}{\partial \xi^{2}}-\xi \frac{\partial \psi_{n}^{\prime \prime}}{\partial \eta}=-\frac{\partial^{2} \psi_{n-1}^{\prime \prime}}{\partial \eta^{2}}, \quad n \geqq 1
$$

where $0<\xi<a \varepsilon^{-1 / 3}, 0<\eta<1$.

The boundary and matching conditions that are applied to the leading interior layer approximation are

$$
\left.\begin{array}{l}
\psi_{0}^{\mathrm{II}}(\xi, \eta)--A \eta \xi^{-1}+O\left(\eta^{2} \xi^{-4}\right), \quad \varepsilon \rightarrow 0, \quad x>0, \\
\psi_{0}^{\mathrm{II}}(\xi, 0)=0 \\
\psi_{0}^{\mathrm{II}}(0, \eta)=f(\eta)
\end{array}\right\}
$$

where the first condition matches the interior layer and outer solutions, and $f(\eta)$ is to be determined so that $\psi$ is continuously differentiable at $x=0$.

By solving (2.6a) subject to $(2.7), \psi_{0}^{\text {"I }}(\xi, \eta)$ is found to be

$$
\psi_{0}^{\mathrm{ll}}(\xi, \eta)=\psi_{1}+\psi_{2}+\psi_{3}
$$

where

$$
\begin{aligned}
\psi_{1}(\xi) & =\frac{1}{2} A \xi^{2} \\
\psi_{2}(\xi, \eta) & =c_{1} \xi \eta^{1 / 3} M\left(-\frac{1}{3}, \frac{4}{3},-\xi^{3} / 9 \eta\right) \\
\psi_{3}(\xi, \eta) & =c_{2} \int_{0}^{\eta} f(u) \xi(\eta-u)^{-4 / 3} \exp \left\{-\xi^{3} / 9(\eta-u)\right\} d u .
\end{aligned}
$$

In this solution, the constants $c_{1}$ and $c_{2}$ are

$$
c_{1}=-A \Gamma\left(\frac{5}{3}\right) 3^{2 / 3}\left\{2 \Gamma\left(\frac{4}{3}\right)\right\}^{-1}, \quad c_{2}=\left\{3^{2 / 3} \Gamma\left(\frac{1}{3}\right)\right\}
$$

and $M$ is Kummer's confluent hypergeometric function. Some elementary substitutions and use of (2.1) then yields an integral equation for $f^{\prime}(\eta)$,

$$
\begin{array}{r}
\int_{0}^{1} f^{\prime}(u) \operatorname{sgn}(\eta-u)|\eta-u|^{-1 / 3} d u=\frac{1}{3} \frac{c_{1}}{c_{2}}\left\{\eta^{1 / 3}+(1-\eta)^{1 / 3}\right\} \\
0<\eta<1,
\end{array}
$$

if $\psi_{0}^{\mathrm{II}}(\xi, \eta)$ is to be continuously differentiable at $x=0$.

The solution,

$$
\begin{aligned}
& f(\eta)=(2 \pi)^{-1} \sin \frac{\pi}{3} \int_{0}^{\eta} R(t)(\eta-t)^{-2 / 3} d t \\
& +\pi^{-2} \sin ^{2} \frac{\pi}{6} \int_{0}^{\eta} t^{-1 / 6}(1-t)^{-1 / 6}(\eta-t)^{-2 / 3}\left\{(P) \int_{0}^{1} \frac{\tau^{1 / 6}(1-\tau)^{1 / 6} R(\tau)}{\tau-t} d \tau\right\} d t \\
& R(\eta)=\frac{1}{3} \frac{c_{1}}{c_{2}}\left\{\eta^{1 / 3}+(1-\eta)^{1 / 3}\right\},
\end{aligned}
$$


of the integral equation (2.10a) may be obtained using a modification of a technique described by Carrier et al [9: p. 426]. Some details of a numerical solution of $(2.10 \mathrm{a})$ have also been given by the author $[3,5]$.

Now it follows from (2.7) that $\psi_{0}^{\text {II }}$ has the property

$$
\varepsilon^{2 / 3} \psi_{0}^{\prime \prime}(\xi, \eta) \sim-\varepsilon^{2 / 3} A \eta\left(x \varepsilon^{-1 / 3}\right)^{-1}+O\left\{\varepsilon^{2 / 3} \eta^{2}\left(x \varepsilon^{-1 / 3}\right)^{-4}\right\}
$$

as $\varepsilon \rightarrow 0$ with $x>0$, or

$$
\lim _{\text {outer }} \varepsilon^{2 / 3} \psi_{0}^{11}(\xi, \eta)=-\varepsilon A\left(y+\frac{1}{2}\right) x^{-1} .
$$

Thus a uniform leading approximation in regions I and II is, simply,

$$
\psi=\varepsilon^{2 / 3} \psi_{0}^{\mathrm{II}}(\xi, \eta)
$$

This term does not necessarily satisfy the boundary conditions at $\gamma_{2}$ and $\gamma_{3}$, that is, the approximation requires additional boundary layers in regions III and IV.

\section{Ordinary boundary layer}

The boundary value $\phi^{*}$ is satisfied at $\gamma_{3}$ if $(2.11)$ is augmented by the boundary layer term $\psi^{\prime \prime \prime}(x, y, \varepsilon)$,

$$
\psi^{111}(x, y, \varepsilon)=\varepsilon b_{3}^{*}(x) e^{-x t}
$$

where, from (1.3),

$$
b_{3}^{*}(x)=b_{3}(x)-\varepsilon^{-1 / 3} \psi_{0}^{\mathrm{I1}}(\xi, 1), \quad 0 \leqq \xi<a \varepsilon^{-1 / 3}
$$

and

$$
t=\left(\frac{1}{2}-y\right) \varepsilon^{-1}
$$

\section{Parabolic boundary layer}

The boundary layer adjacent to $\gamma_{2}$ also has a well known structure (see Eckhaus \& de Jager [14]). It is found that the boundary value $\phi^{*}$ is satisfied at $\gamma_{2}$ if $(2.11)$ is augmented by $\psi^{\text {iv }}(x, y, \varepsilon)$,

$$
\psi^{\prime v}(x, y, \varepsilon)=\varepsilon \frac{2}{\sqrt{\pi}} \int_{(1 / 2) a^{1 / 2} s \eta^{-1 / 2}}^{\infty} b_{2}^{*}\left(\eta-\frac{1}{4} a s^{2} v^{-2}\right) \exp \left(-v^{2}\right) d v
$$

where, from (1.3) and (2.7),

$$
b_{2}^{*}\left(y+\frac{1}{2}\right)=b_{2}(y)+A\left(y+\frac{1}{2}\right) a^{-1}, \quad-\frac{1}{2}<y<\frac{1}{2},
$$

and

$$
s=(a-x) \varepsilon^{-1 / 2}
$$


A simple restriction on the boundary value function $\phi^{*}$ is now introduced to reduce the following analysis. First, the property

$$
b_{2}\left(-\frac{1}{2}\right)=0
$$

is obviously necessary if $\phi^{*}$ is to be continuous, and it follows from (2.14, 2.16) that

$$
\frac{\partial^{2} \psi^{1 \mathrm{v}}}{\partial y^{2}}=\varepsilon\left\{-\frac{1}{4} a^{\frac{1}{2}} s \eta^{-3 / 2} b_{2}^{* \prime}(0)+\frac{2}{\sqrt{\pi}} \int_{(1 / 2) a^{1 / 2} s \eta^{-1 / 2}}^{\infty} b^{* \prime \prime}\left(-\frac{1}{4} a s^{2} v^{-2}\right) e^{-v^{2}} d v\right\} .
$$

Clearly if $b_{2}^{* \prime}(0)=0$ or, from (2.15),

$$
b_{2}^{\prime}\left(-\frac{1}{2}\right)=-A / a
$$

the difficulties associated with the rectangular domain will be reduced. (The property (2.17) precludes the need for regularization of the parabolic boundary layer, as described in [14]).

It is now shown that the confluence of the boundary layer regions III and IV does not cause serious difficulties. First, the function $b_{3}^{*}(x)$ in $(2.13)$ is adjusted so that

$$
\varepsilon b_{3}^{*}(x)=\varepsilon b_{3}(x)-\varepsilon^{2 / 3} \psi_{0}^{\prime \prime}(\xi, 1)-\psi^{\mathrm{iv}}\left(x, \frac{1}{2}, \varepsilon\right)
$$

$\left(0 \leqq \xi<a \varepsilon^{-1 / 3}\right)$ to allow for the $O\left(\varepsilon^{\frac{1}{2}}\right)$ parabolic boundary layer, (see figure 2). This implies that $b_{3}^{*}(x)$ is modified by the parabolic boundary layer in an $O\left(\varepsilon^{\frac{1}{2}}\right)$ neighbourhood of $x=a$. Next, we change $b_{2}^{*}$ to

$$
b_{2}^{*}\left(y+\frac{1}{2}\right)=b_{2}(y)+A\left(y+\frac{1}{2}\right) a^{-1}-b_{3}^{*}(a) e^{-a t},
$$

$\left(-\frac{1}{2}<y<\frac{1}{2}\right)$ to account for the ordinary $O(\varepsilon)$ boundary layer. It follows that $b_{2}^{*}(y)$ is modified only in an $O(\varepsilon)$ neighbourhood of $y=\frac{1}{2}$ and (2.14) then implies that any further adjustments in $b_{2}^{*}$ or $b_{3}^{*}$ are exponentially small as $\varepsilon \rightarrow 0$.

Thus a leading approximation $\psi$ to the solution $\phi$ of $(1.1,1.2)$ is

$$
\psi(x, y, \varepsilon)=\varepsilon^{2 / 3} \psi_{0}^{\prime \prime}(\xi, \eta)+\psi^{\prime \prime \prime}(x, y, \varepsilon)+\psi^{\prime v}(x, y, \varepsilon),
$$

and, in the outer region, this is asymptotic to

$$
\psi(x, y, \varepsilon)=\varepsilon \psi_{0}^{\prime}(x, y)+\psi^{\prime \prime \prime}(x, y, \varepsilon)+\psi^{\mathrm{IV}}(x, y, \varepsilon) .
$$

\section{Pointwise estimation of the error}

\subsection{Preliminaries}

An estimate for the error $w$ in the approximate solution is deduced in $\$ 3.2$ using a technique described elsewhere by the author [4]. A modification 
of the approximation $\psi$ is required before making the estimation, however, since $\psi$ is not continuously differentiable at the points $\left(0, \pm \frac{1}{2}\right)$. The singularities in the derivatives of $\psi$ are discussed in appendix A.

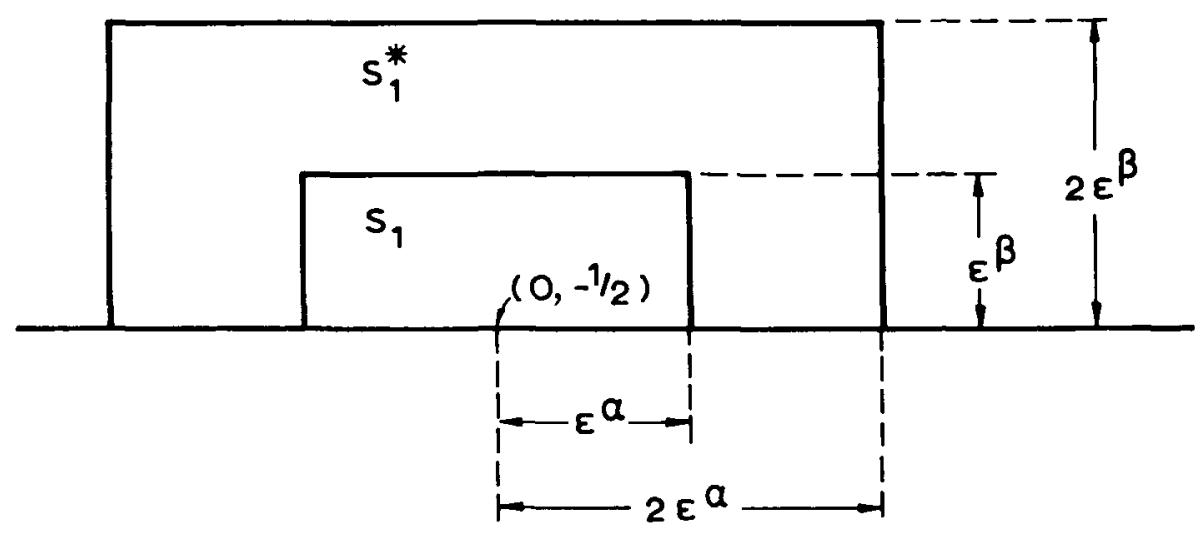

Figure 3.

The strip $S_{1}$ and the border $S_{1}^{*}$.

First, consider neighbourhoods of the point $\left(0,-\frac{1}{2}\right)$ and introduce a $C^{\infty}(\bar{G})$ mollifier which annihilates functions in a small rectangular strip $S_{1}$. Let $m_{1}(x, y)$ be a $C^{\infty}(\bar{G})$ function in the border $S_{1}^{*}$ surrounding $S_{1}$ (see figure 3) with the properties

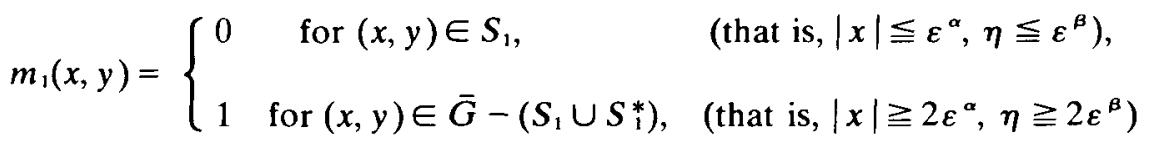

in the adjoining regions. Such a mollifier must satisfy

$$
\nabla m_{1}=\mathbf{0} \text { at } \partial S_{1}^{*}
$$

where $S_{1}^{*}$ is the boundary of the border region $S_{1}^{*}$.

A second mollifier $m_{2}(x, y)$ may be defined to exclude the corresponding rectangular strip $S_{2}$ containing $\left(0, \frac{1}{2}\right)$. The precise size of the strips $S_{1}$ and $S_{2}$ are established in appendix B.

These mollifiers enable a continuously differentiable approximation

$$
\psi^{*}=\varepsilon^{2 / 3} \psi_{0}^{\mathrm{II}}(\xi, \eta) m_{1}(x, y) m_{2}(x, y)+\psi^{\mathrm{II \prime}}(x, y, \varepsilon)+\psi^{\mathrm{IV}}(x, y, \varepsilon)
$$

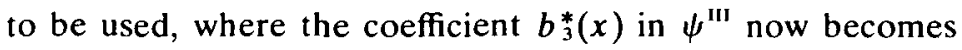

$$
\varepsilon b_{3}^{*}(x)=\varepsilon b_{3}(x)-m_{1}(x, y) m_{2}(x, y)\left\{\varepsilon^{2 / 3} \psi_{0}^{\mathrm{II}}(\xi, 1)\right\}-\psi^{\mathrm{iv}}\left(x, \frac{1}{2}, \varepsilon\right)
$$

in order that $\psi^{*}$ satisfies the boundary value (1.3). 
An inequality relating the norms \|\|$_{w_{2}^{1}(G)}$ and \|\|$_{C(G)}$

In the following lemma, $D$ is a closed domain, $(x, y)$ and $\left(x^{\prime}, y^{\prime}\right)$ are general points in $D$, and $\zeta(r)$ denotes any $C^{\infty}$ function with

$$
\zeta(r)= \begin{cases}1 & r \leqq \frac{1}{2} R \\ 0 & r \geqq R\end{cases}
$$

where $0<R<\operatorname{diam} . D, r=\left|(x, y)-\left(x^{\prime}, y^{\prime}\right)\right|$.

LEMMA [4] Let $u(x, y)$ be a continuously differentiable function defined on a closed domain $D$ which possesses the cone property. Then the function $u$ satisfies the following relations:

I If $u$ and its derivatives are sufficiently small such that

$$
\sup _{D} \ln \left|\frac{\partial}{\partial r}(\zeta u)\right| \leqq 1,
$$

then for any $p$ in the interval $1<p<2$, and $(x, y)$ in $D$,

$$
u(x, y) \leqq c_{1}(2-p)^{-1 / p}\left\{\iint_{D}\left(u^{2}+(\nabla u)^{2}\right) d x d y\right\}^{(p-1) / p}
$$

II Alternatively, if

$$
\sup _{D} \ln \left|\frac{\partial}{\partial r}(\zeta u)\right|>1,
$$

there exists $q$ in the interval $1<q<2$ such that for any $(x, y)$ in $D$,

$$
|u(x, y)| \leqq c_{2}\left(c_{3}+\sup _{D} \ln \left(u^{2}+(\nabla u)^{2}\right)\right)^{1 / q}\left\{\iint\left(u^{2}+(\nabla u)^{2}\right) d x d y\right\}^{(q-1) / q}
$$

The constants $c_{1}, c_{2}, c_{3}$ are independent of $u$ and it is shown in reference [4] that the index $q$ is defined by

$$
(2-q) \sup _{D} \ln \left|\frac{\partial}{\partial r}(\zeta u)\right|=1
$$

Estimation of the norm \|\|$_{w_{2}^{1}(G)}$

The error $w=\phi-\psi^{*}$ satisfies the elliptic boundary value problem

$$
\begin{gathered}
\varepsilon \nabla^{2} w-x \frac{\partial w}{\partial y}=\varepsilon g(x, y, \varepsilon) \text { in } G . \\
\left.z\right|_{\partial G}=0
\end{gathered}
$$

where the term $\varepsilon g$ is defined in appendix B. These equations may be used to obtain a standard energy estimate for $w$, 


$$
\begin{aligned}
\|w\|_{w_{2}^{\prime}(G)} & =\left\{\iint_{G}\left(w^{2}+(\nabla w)^{2}\right) d x d y\right\}^{1 / 2} \\
& \leqq \sqrt{2}\left\{\iint_{G}|w g| d x d y\right\}^{1 / 2}
\end{aligned}
$$

\subsection{Estimation of the error}

If the second alternative in the above Lemma applies, equations (3.4) and (3.7) imply

$$
|w(x, y)| \leqq c_{2} 2^{(q-1) / q}\left\{c_{3}+\sup _{G} \ln \left(w^{2}+(\nabla w)^{2}\right)\right\}^{1 / q}\left\{\iint_{G}|w g| d S\right\}^{(q-1) / q}
$$

whence

$$
\sup _{G}|w(x, y)| \leqq c_{2}^{q} 2^{q-1}\left\{c_{3}+\sup _{G} \ln \left(w^{2}+(\nabla w)^{2}\right)\right\}\left\{\iint|g| d S\right\}^{q-1}
$$

To estimate the logarithmic term in (3.8), we observe that the term $\varepsilon g$ in (3.6a) has the bound

$$
\sup _{G}|\varepsilon g|<c_{4} \varepsilon^{-\nu} \quad \text { for some fixed } \nu<\infty,
$$

and the boundary data (3.6b) and Schauder's estimates (see for example, Courant \& Hilbert [12: p. 336]) then yield ${ }^{\dagger}$

$$
\sup _{G}\left(w^{2}+(\nabla w)^{2}\right)=c_{s} \varepsilon^{-\mu} \quad \text { for fixed } \mu<\infty .
$$

Also, (3.5) gives for the index $q$

$$
q=2-c_{6}|\ln \varepsilon|^{-1}+O\left(|\ln \varepsilon|^{-2}\right)
$$

where $c_{6}>0$ is independent of $\varepsilon$. Therefore, the inequalities $(3.8,3.10)$ give the error bound

$$
\sup _{G}|w| \leqq c_{7}|\ln \varepsilon|\left\{\iint_{G}|g| d S\right\}^{q-1}
$$

'This point requires clarification for, in the cited reference, Schauder's estimates are presented for a domain with a smooth boundary. The author is unaware of estimates in which the smooth boundary requirement is relaxed, possibly a conformal map could be used for this purpose. Schauder's estimates should still be applicable, however, for the rectangular boundary assumed for this problem in view. of the smooth boundary values and the mildness of the required bound (3.9). 
where $q$ is given by (3.10) and $c_{7}$ is a positive constant independent of $\varepsilon$ and $w$.

The integral in (3.11) is now estimated and, for this purpose, the domain $G$ is divided into several regions for $x>0$ (see figure 2), that is,

the interior layer $\left\{(x, y)|| x \mid \leqq k_{1} \varepsilon^{1 / 3}, \quad-\frac{1}{2}+k_{2} \leqq y \leqq \frac{1}{2}-k_{2}\right\}$,

the outer region $\left\{(x, y)|| x \mid>k_{1} \varepsilon^{1 / 3}, \quad-\frac{1}{2}<y<\frac{1}{2}\right\}$,

the corner regions $\left\{(x, y)|| x \mid \leqq k_{1} \varepsilon^{1 / 3},-\frac{1}{2}<y<-\frac{1}{2}+k_{2}\right.$ or $\left.\frac{1}{2}-k_{2}<y<\frac{1}{2}\right\}$.

Here, $k_{1}$ and $k_{2}$ are constants independent of $\varepsilon$ with $k_{2} \ll 1$. Also the inhomogeneous term $\varepsilon g$ in (3.6a) is decomposed into two terms $\varepsilon g_{1}+\varepsilon g_{2}$ where, from the defining equations $(B \cdot 1 b, B \cdot 1 c)$,

$$
\begin{aligned}
\varepsilon g_{1}= & \varepsilon A(1-m)-\varepsilon m \frac{\partial^{2}}{\partial y^{2}}\left\{\varepsilon^{2 / 3} \psi_{0}^{11}(\varepsilon, \eta)\right\} \\
& + \text { terms involving derivatives of } m_{1}(x, y) \text { and } m_{2}(x, y), \\
\varepsilon g_{2}= & -\varepsilon \frac{\partial^{2}}{\partial x^{2}}\left\{\psi^{\prime \prime \prime}(x, y, \varepsilon)\right\}-\varepsilon \frac{\partial^{2}}{\partial y^{2}}\left\{\psi^{\mathrm{IV}}(x, y, \varepsilon)\right\}
\end{aligned}
$$

(The term $m$ denotes $m_{1}(x, y) m_{2}(x, y)$ )

The construction of the above functions leads to the bounds

$$
\max _{\text {interiorlayer }}\left|g_{1}\right|<c \varepsilon^{2 / 3}, \quad \sup _{\text {outer region }}\left|g_{1}\right|<c \varepsilon /|x|
$$

where $c$ is a constant depending on $k_{1}$ and $k_{2}$, and it readily follows that

$$
\iint_{\text {interiorlayer }}\left|g_{1}\right| d S=O(\varepsilon), \iint_{\text {outer region }}\left|g_{1}\right| d S=O(\varepsilon|\ln \varepsilon|) .
$$

Further, equations $(2.12,2.14,2.19,3.2)$ can be used to show

$$
\iint_{G}\left|g_{2}\right| d S=O(\varepsilon)
$$

and finally, it is shown in appendix B that a suitable choice for the mollifier parameters $\alpha$ and $\beta$ leads to the estimate

$$
\iint_{\text {corner regions }}\left|g_{1}\right| d S=O\left(\varepsilon^{\frac{2}{3+\delta}}\right)
$$

where $\delta$ is a small positive constant.

Substituting the estimates (3.12-3.14) into (3.11) therefore yields the pointwise bound

$$
\sup _{G}|w|=O\left(|\ln \varepsilon| \varepsilon^{\frac{12}{3}+\delta K 1-c_{\mathrm{o}}|\ln \varepsilon|^{-1}+O\left(|\ln \varepsilon|^{-2}\right)}\right), \quad \varepsilon \rightarrow 0,
$$


which reduces to

since

$$
\sup _{\sigma}|w|=O\left(|\ln \varepsilon| \varepsilon^{\frac{2}{3}+\delta}\right), \quad \varepsilon \rightarrow 0
$$

$$
\varepsilon^{-k|i n \varepsilon|^{-1}}=\exp (-k)
$$

is independent of $\varepsilon$.

Equation (3.15) shows that $\psi^{*}$ is asymptotic to $\phi$ in the interior layer (although only by a narrow margin), that is in the interior layer,

$$
\left|\phi-\psi^{*}\right|=o\left(\psi^{*}\right) \text { as } \varepsilon \rightarrow 0, \quad 0<\varepsilon<\varepsilon_{0} \ll 1 .
$$

In the remaining regions of $G$, however, $\left|\phi-\psi^{*}\right|$ is not $o\left(\psi^{*}\right)$ as $\varepsilon \rightarrow 0$. Clearly the result (3.15) would be sharpened if a better approximation were constructed in the corner regions since the mollifying technique is a crude way of obtaining a continuously differentiable approximation. The derivation of sharper estimates is discussed in the next section.

The above analysis has been based on the assumption that the second alternative of the Lemma applies. A similar analysis can be used if the first alternative holds, thus giving the error bound

$$
\sup _{G}|w| \leqq \text { const. }(2-p)^{-1} \varepsilon^{\left(\frac{2}{3}+\delta\right)(p-1)}, \quad 1<p<2,
$$

whence the asymptotic property (3.16) still holds provided

$$
p-1>\left(1+\frac{3}{2} \delta\right)^{-1}
$$

The estimate (3.17) may be made sharper than (3.15) by a suitable choice for the index $p$.

\section{Improved error estimates}

The error estimates derived above are useful only in the interior layer since, elsewhere in the domain, the approximation $\psi^{*}$ has a smaller magnitude than the error bound. Further, although the number $\delta$ in $(3.15,3.17)$ has not been specified precisely, an examination of the estimates from which it is derived (equations B.2) shows that $\delta$ could not be larger than about 0.05 . Thus the error bounds are certainly not sharp. In this section, it is described how $\psi^{*}$ might be proved asymptotic to $\phi$ in the outer region as $\varepsilon \rightarrow 0$, and a procedure which would establish the asymptoticity of higher approximations is outlined.

It is clear that the accuracy of the outer approximation can be established only by calculating better approximations or by using some other estimation technique. Certainly a better estimate would result from better mollifiers, but 
even if the contributions of the corner region to the estimate were negligible, the theory would still give an unsatisfactory bound,

$$
\sup _{G}|w|=O\left(\varepsilon|\ln \varepsilon|^{2}\right)
$$

The accuracy of approximate solutions for certain related singular perturbation problems has been examined by Eckhaus \& de Jager [14] using the maximum principle for elliptic equations. For the present problem, their results become applicable away from the interior layer. Thus if sub-domains in which $|x|>l$ are considered ( $l$ is $O(1)$ as $\varepsilon \rightarrow 0$ ), Eckhaus \& de Jager's analysis implies a "barrier" or dominating function for $w$ can be defined to show

$$
\left|\phi-\psi^{*}\right|=o\left(\psi^{*}\right), \quad|x|>l+O\left(\varepsilon^{\frac{1}{2}}\right), \quad \varepsilon \rightarrow 0 .
$$

That is, if the sub-domain $|x|>l$ is considered to be separate from the interior layer, the outer solution is still determined by the boundary conditions applied at $\gamma_{1}$ and $\gamma_{4}$. Further, the effect of a changed boundary condition at $|x|=l$ is restricted to an $O\left(\varepsilon^{\frac{1}{2}}\right)$ parabolic boundary layer along $|x|=l$.

Therefore, $\psi^{*}$ is known to be asymptotic to $\phi$ except in the narrow transition region between the interior layer and the sub-domain with $|x|>$ $l+O\left(\varepsilon^{\frac{1}{2}}\right)$.

We now consider the possibility of deriving general results. Suppose a function $\psi_{N}(x, y, \varepsilon)$ with the generalized asymptotic expansion

$$
\psi_{N}(x, y, \varepsilon)=\sum_{n=0}^{N} \delta_{n}(\varepsilon) \psi_{N}(x, y, \varepsilon)
$$

were to be constructed, where $\left\{\delta_{n}\right\}_{n=0}^{\infty}$ is an asymptotic sequence and $\psi_{N}$ is a formal approximation to $\phi$ in the sense that

$$
\begin{gathered}
\varepsilon \nabla^{2} \psi_{N}-x \frac{\partial \psi_{N}}{\partial y}=\varepsilon A+O\left(\delta_{N+1}(\varepsilon)\right) \text { in } G, \\
\left.\psi_{N}\right|_{\partial G}=\phi^{*}
\end{gathered}
$$

The construction of such an approximation is a daunting prospect for the present problem in view of various boundary and interior layer regions. The theory of $\$ 3$ then gives an error bound on $w_{N}=\phi-\psi_{N}$,

$$
\sup _{\sigma}\left|w_{N}\right|=O\left(|\ln \varepsilon| \varepsilon^{-1} \delta_{N+1}(\varepsilon)\right) .
$$

Since two approximations $\psi_{N}$ and $\psi_{M}(M<N)$ differ only by terms which are $o\left(\psi_{M}\right)$ uniformly as $\varepsilon \rightarrow 0,(4.4)$ proves the asymptoticity of $\psi_{M}$ where $M$ is an index such that 


$$
|\ln \varepsilon| \varepsilon^{-1} \delta_{N+1}(\varepsilon)=o\left(\delta_{M}(\varepsilon)\right) \quad \text { as } \quad \varepsilon \rightarrow 0 .
$$

The theory described above is based on an a priori estimate [4] for elliptic equations in two variables. For higher dimensions, there are general a priori estimates available [2]. In the present case, however, the use of general estimates would lead to inferior results to the above.

\section{Acknowledgements}

The author would like to acknowledge the generous assistance of Dr. P. B. Chapman and Professor J. J. Mahony. This paper was taken from the author's $\mathrm{Ph}$. D. thesis [3] and it was partly written during the tenure of a CSIRO Postdoctoral Studentship at the University of Cambridge.

\section{Appendix A}

Bounds for the derivatives $\partial^{2} \psi_{2} / \partial \eta^{2}$ and $\partial^{2} \psi_{3} / \partial \eta^{2}$ are established in this appendix.

The $\eta$-derivatives of $\psi_{2}(\xi, \eta)$ may be obtained by direct differentiation and the properties of Kummer's function $M\left(-\frac{1}{3}, \frac{4}{3},-x^{3} /(9 \varepsilon \eta)\right.$ ) (see, for example [1]). Introducing $k$,

$$
k=-x^{3} /(9 \varepsilon \eta)
$$

we obtain for $k$ moderate and large

$$
\left|\varepsilon^{2 / 3} \frac{\partial^{2} \psi_{2}}{\partial \eta^{2}}\right| \leqq \begin{cases}\hat{c}_{1} \varepsilon^{1 / 3} x \eta^{-5 / 3} & \text { for }|k| \leqq \hat{k} \\ \hat{c}_{2} \varepsilon^{2} x^{-4} & \text { for }|k|>\hat{k}\end{cases}
$$

Here $\hat{k}, \hat{c}_{1}$ and $\hat{c}_{2}$ are $O(1)$ constants, and $\psi_{2}(\xi, \eta)$ is singular only near $x=0$, $y=-\frac{1}{2}$ for $x \geqq 0$.

A discussion of $\psi_{3}(\xi, \eta)$ given by (2.8d) requires a knowledge of $f(\eta)$ as $\eta \rightarrow 0$ and $\eta \rightarrow 1$. Evaluating the principal value integrals in (2.10b) using a table of Hilbert transforms and estimating the resultant for $\eta$ small gives

$$
2 f(\eta) / A=-2.35 \eta^{1 / 6}-1.43 \eta^{2 / 3}+1.28 \eta^{7 / 6}+\cdots, \quad 0<\eta<\eta_{0} \ll 1
$$

The behaviour of $f(\eta)$ as $\eta \rightarrow 1$ is given by symmetry.

The estimation of the derivatives of $\psi_{3}$ may now be completed by differentiation and direct substitution. A lengthy, but straightforward calculation shows that near $x=0, y=-\frac{1}{2}$, and for $k_{1}=x^{3} /(9 \varepsilon \eta)$ moderate and large,

$$
\left|\varepsilon^{2 / 3} \frac{\partial^{2} \psi_{3}}{\partial \eta^{2}}\right| \leqq \begin{cases}\bar{c}_{1} \varepsilon^{2 / 3} \eta^{-11 / 6} & \text { for } 0 \leqq k_{1} \leqq \bar{k}_{1} \\ \tilde{c}_{2} \varepsilon^{2 / 3} \eta^{-11 / 6} \exp \left(-k_{1}\right) & \text { for } k_{1}>\bar{k}_{1} .\end{cases}
$$


Similarly, near $x=0, y=\frac{1}{2}$ and for $k_{2}=x^{3} / 9 \varepsilon(1-\eta)$ moderate and large,

$$
\left|\varepsilon^{2 / 3} \frac{\partial^{2} \psi_{3}}{\partial \eta^{2}}\right| \leqq \begin{cases}\bar{c}_{1} \varepsilon^{2 / 3}(1-\eta)^{-11 / 6} & \text { for } 0 \leqq k_{2} \leqq \bar{k}_{2} \\ \bar{c}_{2} \varepsilon^{5 / 2} x^{-11 / 2} & \text { for } k_{2}>\bar{k}_{2} .\end{cases}
$$

All the constants in (A.3, A.4) are independent of $\varepsilon$ and corresponding results for $x<0$ are given by symmetry.

\section{Appendix B}

The mathematical steps that are required to establish the estimate (3.14) are described in this appendix. In full, the elliptic partial differential equation for the error $w$ is

$$
\varepsilon \nabla^{2} w-x \frac{\partial w}{\partial y}=\varepsilon g_{1}+\varepsilon g_{2}
$$

where

$$
\begin{aligned}
\varepsilon g_{1}= & \varepsilon A(1-m)-\varepsilon m \frac{\partial^{2}}{\partial y^{2}}\left\{\varepsilon^{2 / 3} \psi_{0}^{\prime \prime}(\xi, \eta)\right\}-\varepsilon \nabla \cdot\left[\varepsilon^{2 / 3} \psi_{0}^{\prime \prime}(\xi, \eta) \nabla m\right] \\
& -\varepsilon \nabla m \cdot \nabla\left[\varepsilon^{2 / 3} \psi_{0}^{\prime \prime}(\xi, \eta)\right]+x \varepsilon^{2 / 3} \psi_{0}^{\prime \prime \prime}(\xi, \eta) \frac{\partial m}{\partial y} \\
\varepsilon g_{2}= & -\varepsilon \frac{\partial^{2}}{\partial x^{2}}\left\{\psi^{\prime \prime \prime}(x, y, \varepsilon)\right\}-\varepsilon \frac{\partial^{2}}{\partial y^{2}}\left\{\psi^{\mathrm{IV}}(x, y, \varepsilon)\right\} .
\end{aligned}
$$

(Again, $m$ denotes the mollifier product $m_{1}(x, y) m_{2}(x, y)$. Only neighborhoods of the point $x=0, y=-\frac{1}{2}$ are considered here as the contribution from the other corner region is identical.

The estimate is obtained by choosing the mollifier parameters $\alpha$ and $\beta$ so that the contributions from all the non-homogeneous terms of equation (B.1a) are sufficiently small. For this purpose, the singularities in these terms have been determined in appendix $A$, and reference is made to the estimates (A.2-A.4) in which the constants denoted by $\left.\left(^{(}\right),()^{\circ}\right),\left(^{-}\right)$are of $O(1)$ as $\varepsilon \rightarrow 0$.

Suppose the parameters $\alpha$ and $\beta$ are chosen so that the configuration depicted in figure 4 applies, and let the regions $R_{1}, R_{2}$ and $R_{3}$ be as shown. The situation sketched in figure 4 holds provided

$$
(1+\beta) / 3 \geqq \alpha>\frac{1}{3}
$$

and the admissible values of $\alpha$ and $\beta$ are illustrated in figure 5 . 


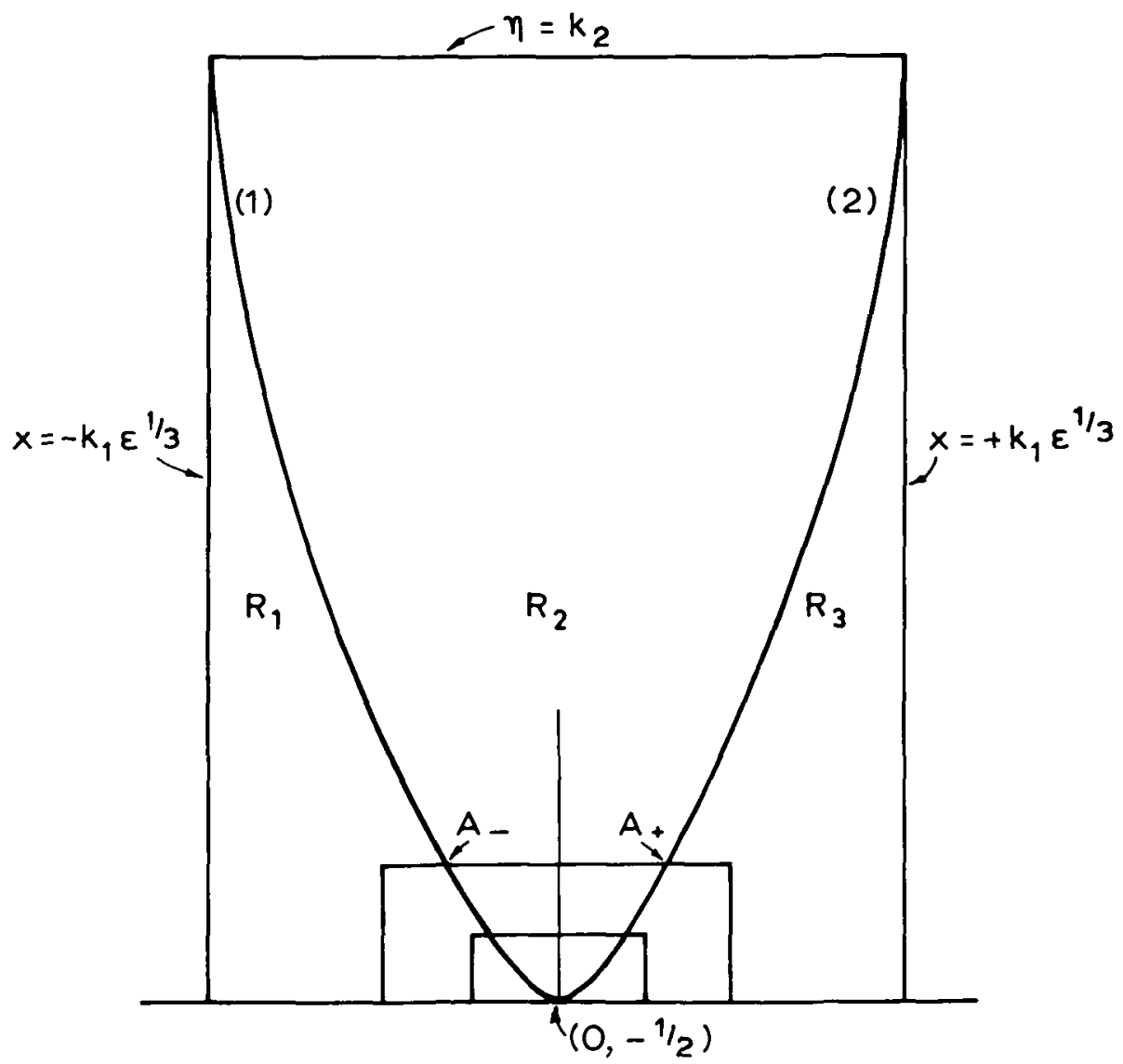

Figure 4.

The configuration in the corner region. The curves (1) and (2) are given by $\left|x^{3} /(9 \varepsilon \eta)\right|=\bar{k}$ and $\left|x^{3} /(9 \varepsilon \eta)\right|=\hat{k}$ (or $\left.\tilde{k}\right)$. The points $A_{-}$and $A_{+}$are

$$
A_{-}=\left(-(18 \bar{k})^{1 / 3} \varepsilon^{(1+\beta) / 3}, 2 \varepsilon^{3}\right), \quad A_{+}=\left((18 \hat{k})^{1 / 3} \varepsilon^{(1+\alpha) / 3}, 2 \varepsilon^{B}\right)
$$

The contributions to $\iint_{\text {connerregions }}\left|g_{1}\right| d S$ from each of the nonhomogeneous terms of equation (B.1a) are

$$
\begin{aligned}
& \iint_{\text {conner regions }} A(1-m)=O\left(\varepsilon^{\alpha+\beta}\right), \\
& \iint_{R_{1}} m \frac{\partial^{2}}{\partial y^{2}}\left\{\varepsilon^{2 / 3} \psi_{0}^{\prime \prime}\right\} d S=O\left(\varepsilon^{\min \left(\frac{3}{2}-\frac{3}{2} \alpha, 1\right\}}\right), \\
& \iint_{R_{2}} m \frac{\partial^{2}}{\partial y^{2}}\left\{\varepsilon^{2 / 3} \psi_{0}^{\prime \prime}\right\} d S=O\left(\varepsilon|\ln \varepsilon|, \varepsilon^{1-\frac{1}{2} \beta}\right),
\end{aligned}
$$




$$
\begin{aligned}
& \iint_{R_{3}} m \frac{\partial^{2}}{\partial y^{2}}\left\{\varepsilon^{2 / 3} \psi_{0}^{11}\right\} d S=O\left(\varepsilon|\ln \varepsilon|, \varepsilon^{1-\frac{1}{2} \beta}\right), \\
& \iint_{S_{1}} \nabla \cdot\left(\varepsilon^{2 / 3} \psi_{0}^{\prime \prime} \nabla m\right) d S=0 \\
& \iint_{\varsigma_{1}} \nabla m \cdot \nabla\left[\varepsilon^{2 / 3} \psi_{0}^{\prime \prime}\right] d S=O\left(\varepsilon^{\min \left(\frac{1}{3}+\beta .1+2 \beta-2 \alpha, 1-\frac{1}{2} \beta \cdot \frac{3}{2}-\frac{3}{2} \alpha\right\}}\right), \\
& \varepsilon^{-1} \iint_{S_{1}} x \varepsilon^{2 / 3} \psi_{0}^{11} \frac{\partial m}{\partial y} d S=O\left(\varepsilon^{2 \alpha-\frac{1}{3}}\right) .
\end{aligned}
$$

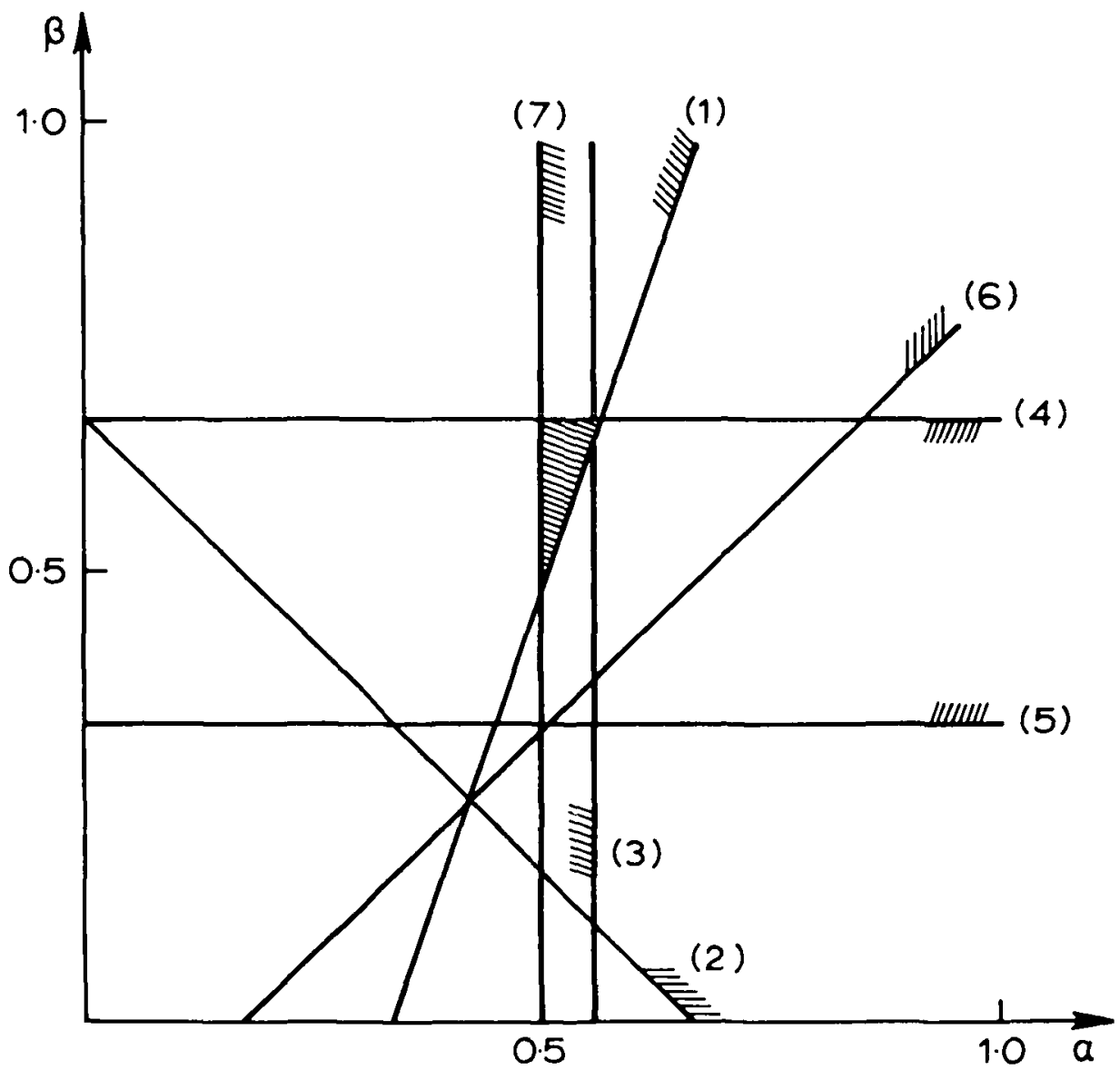

Figure 5 .

Admissible values for the mollifier parameters $\alpha$ and $\beta$. The lines are: (1) $1+\beta=3 \alpha$, (2) $\alpha+\beta=\frac{2}{3}$, (3) $\alpha=\frac{5}{9}$, (4) $\beta=\frac{2}{3}$, (5) $\beta=\frac{1}{3}$ (6) $\alpha-\beta=\frac{1}{6}$, (7) $\alpha=\frac{1}{2}$. 
The above estimates have been established using crude bounds and some of them (particularly (B.2g)) are unduly pessimistic. The reduced domain $S_{1}^{*}$ is used in the last three estimates since the derivatives of $m$ are zero elsewhere.

Now the application of $\S 3$ requires that $\iint_{\text {cornerregions }}\left|g_{1}\right| d S$ be of $O\left(\varepsilon^{\wedge}\right)$ as $\varepsilon \rightarrow 0$ where $\lambda>\frac{2}{3}$. The estimates (B.2a) to (B.2g) above therefore yield the set of inequalities:

$$
\left.\begin{array}{c}
\alpha+\beta>\frac{2}{3}, \alpha<\frac{5}{9}, \beta<\frac{2}{3}, \beta>\frac{1}{3}, \\
\frac{1}{6}-\alpha+\beta>0, \alpha>\frac{1}{2} .
\end{array}\right\}
$$

The admissible values of $\alpha$ and $\beta$ satisfying these inequalities are shown in figure 5 and the property (3.14),

$$
\iint_{\text {conner regions }}\left|g_{1}\right| d S=O\left(\varepsilon^{\frac{2}{3+5}}\right), \quad \delta>0,
$$

holds if the parameters $\alpha$ and $\beta$ lie in the range indicated by the shaded triangle.

\section{References}

[1] M. Abramowitz \& I. A. Stegun, Handbook of mathematical functions, Dover, New York (1965).

[2] S. Agmon, 'The $L_{p}$ approach to the Dinchlet problem' Annali Scu. Norm. Sup. - Pisa, 13 (1959), 405-448.

[3] N. G. Barton, Interior layers in rotating fluids, Ph.D. thesis, University of Western Aust ralia (1973).

[4] N. G. Barton, 'An ınequality for the pointwise estımation of solutions of second order elliptic partial differential equations: J. Inst. Maths Applics, 14 (1974), 325-333.

[5] N. G. Barton, 'A device for the numerical solution of Fredholm integral equations of the first kind', Applied Mathematics Preprint No. 83, Department of Mathematics, University of Queensland (1975).

[6] N. G. Barton, 'An example of the modification of ocean currents by bottom topography', to appear in Tellus, 28 (1976), 261-265.

[7] M. S. Berger \& L. E. Fraenkel, 'On the asymptotic solution of a non-linear Dirichlet problem', J. Math. Mech., 19 (1970), 553-585.

[8] K. H. Brink, G. Veronis \& C. C. Yang, 'The effect on ocean circulation of a change in the sign of $\beta$, Tellus, 25 (1973), 518-521.

[9] G. F. Carrier, M. Krook \& C. E. Pearson, Functions of a complex variable, McGraw-Hill, New York (1966).

[10] L. P. Cook \& G. S. S. Ludford, 'The behaviour as $\varepsilon \rightarrow+0$ of solutions to $\varepsilon \nabla^{2} w=\partial w / \partial y$ in $|y| \leqq 1$ for discontinuous boundary data, SIAM J. Math. Anal., 2 (1971), 567-594.

[11] L. P. Cook, G. S. S. Ludford \& J. S. Walker, 'Corner regions in the asymptotic solution of $\varepsilon \nabla^{2} u=\partial u / \partial y$ with reference to MHD duct flow', Proc. Cam. Phil. Soc., 72 (1972), $117-122$. 
[12] R. Courant \& D. Hilbert, Methods of mathematical physics, Interscience, Vol 2, New York (1962).

[13] J. M. de Villiers, 'A uniform asymptotic expansion of the positive solution of a non-linear Dirichlet problem', Proc. London Math. Soc. (3), 27 (1973), 701-722.

[14] W. Eckhaus \& E. M. de Jager, 'Asymptotic solutions of singular perturbation problems for lınear differential equations of elliptic type', Arch. Ratıonal Mech. Anal. 23 (1966-67), 26-86.

[15] $\dddot{w}$. Eckhaus, 'Boundary layers in linear elliptic singular perturbation problems', SIAM Review, 14 (1972), 225-270.

[16] C. B. Fandry \& L. M. Leslie, 'A note on the effect of latitudinally varying bottom topography on the winddriven ocean circulation', Tellus, 24 (1972), 164-167.

[17] J. A. Johnson, C. B. Fandry \& L. M. Leslie, 'On the variation of ocean circulation produced by bottom topography', Tellus, 23 (1971), 113-122.

[18] V. M. Kamenkovich \& V. A. Mitrofanov, 'An example of ocean-bottom topography on currents', translated from Doklady, Akad. Nauk, SSSR, 199 (1971), 78-81.

School of Mathematics,

University of New South Wales,

Kensington, N.S.W. 2033,

Aust ralia. 